\title{
Perfil Audiológico em Policiais Militares do Estado de São Paulo
}

\author{
Audiology Profile in the Military Police State of São Paulo
}

\author{
Heraldo Lorena Guida*, Thiago Hernandes Diniz**, Patrícia da Silva Carlos Chagas **, \\ Sérgio Koodi Kinoshita***.
}

\author{
* Doutor. Professor Assistente Doutor. \\ *** Bacharel em Fonoaudiologia. Fonoaudiólogo (a). \\ **** Doutor. Professor Doutor. \\ Instituição: Faculdade de Filosofia e Ciências - Departamento de Fonoaudiologia, UNESP - Campus de Marília. \\ Marília / SP - Brasil. \\ Endereço para correspondência: Heraldo Lorena Guida - Avenida Hygino Muzzi Filho, 737 - Caixa Postal 181 - Marília / SP - Brasil - CEP: $17525-900$ - Telefone: \\ (+55 14) 3402-1324 - E-mail: hlguida@marilia.unesp.br \\ Suporte Financeiro: Fundação de Amparo à Pesquisa do Estado de São Paulo - FAPESP (Processo № 08/50720-1). \\ Artigo recebido em 22 de Julho de 2010. Artigo aprovado em 7 de Setembro de 2010
}

\section{RESUMO}

Introdução:

Objetivo:

Método:

Resultados:

Conclusão:

Palavras-chave:

\section{SUMMARY}

Introduction:

Objective:

Method:

Results:

Conclusion:

Keywords:
Os policiais militares constituem uma população exposta a ruído de impacto devido aos treinamentos com armas de fogo.

Pesquisar o perfil audiológico em policiais militares do estado de São Paulo, e correlacionar à idade e o tempo de exposição com os achados audiológicos.

Neste estudo de série de casos, transversal, foi realizada avaliação audiológica em 200 policiais militares sendo 169 (84,5\%) do gênero masculino e 31 (15,5\%) do feminino, com idades entre 25 e 45

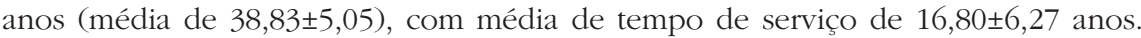

As principais queixas apontadas foram: zumbido $(n=52 / 26 \%)$, disacusia $(n=36 / 18 \%)$, plenitude auricular $(n=24 / 12 \%)$ e autofonia $(n=24 / 12 \%)$. Na imitanciometria foram encontradas 100\% de curvas tipo A (Jerger, 1970), e houve presença de recrutamento em 20 (10\%) casos. Segundo os resultados da audiometria tonal, os dados obtidos demonstraram que 54 (27\%) orelhas direita e 56 (28\%) orelhas esquerda apresentaram perda auditiva. Os graus de perda com maior ocorrência foram 1 e 2 (MerLuzzI et al., 1979), com 85 (42,5\%) casos. Houve correlação significativa entre a idade e o tempo de exposição com a piora dos limiares audiométricos em ambas as orelhas.

A partir da análise dos dados audiológicos foi possível verificar que os policiais militares são uma população que apresenta risco para desenvolver a perda auditiva. Sendo assim, vemos a necessidade de implementação de programa de conservação auditiva para os policiais militares. polícia, ruído ocupacional, perda auditiva.

The military police are a population exposed to noise impact due to training with firearms.

To investigate the audiological profile of the military police in São Paulo state, and to correlate the age and duration of exposure and audiological findings.

In this study of case series, cross-sectional audiological evaluation was performed in 200 police officers being 169 (84.5\%) were male and 31 (15.5\%) females, aged between 25 and 45 years (mean $38.83 \pm$ $5.05)$, average service time of $16.80 \pm 6.27$ years.

The main complains were: tinnitus ( $n=52 / 26 \%)$, hearing loss $(n=36 / 18 \%)$, ear fullness $(n=24 / 12 \%)$ and autophonia ( $n=24 / 12 \%$ ). Impedance were found in 100\% of type A curves (JERGER, 1970), and recruitment was present in 20 (10\%) cases. According to the results of audiometry, the data showed that $54(27 \%)$ right ears and 56 (28\%) left ears had hearing loss. The degree of loss with higher prevalence was 1 and 2 (Merluzzi et al., 1979), 85 (42.5\%) cases. There was significant correlation between age and exposure time with the worsening of audiometric thresholds in both ears.

From the audiological data analysis was possible that the military police are a population that presents a risk to develop hearing loss. Thus, we see the need for implementation of hearing conservation program for the military police.

police, occupational noise, hearing loss 


\section{INTRODUÇÃO}

A exposição ao ruído por um longo período de tempo pode causar desvios importantes ao ser humano, à perda auditiva induzida por ruído (PAIR), se caracteriza por uma alteração dos limiares auditivos, tipo neurossensorial, irreversível e progressiva. A perda auditiva atinge principalmente as células ciliadas da cóclea situadas a cerca de 5 a $10 \mathrm{~mm}$ da janela vestibular, justamente na região receptora dos estímulos de 4 a $6 \mathrm{kHz}$ (1). A PAIR acomete inicialmente a faixa de frequência entre 3 e $6 \mathrm{kHz}$, onde o limiar de $8 \mathrm{kHz}$ tem de estar melhor que o pior limiar (3, 4 ou $6 \mathrm{kHz})(2,3)$.

Alguns estudos verificaram que a frequência de 6 $\mathrm{kHz}$ é a mais acometida nas audiometrias sugestivas de PAIR ( $4,5,6)$, enquanto outros estudos, referiram que a frequência de $4 \mathrm{kHz}$ é a mais comprometida $(7,8,9)$.

O zumbido e a hipoacusia são queixas importantes relacionadas à PAIR citadas na literatura $(10,11)$. Em um estudo realizado para avaliar a prevalência e o grau de perda auditiva induzida por ruído em militares de carreira, foram diagnosticas perda auditiva em $68 \%(n=475)$ dos casos, destes em $42,5 \%$ houve queixa de zumbido contínuo (12).

A investigação dos efeitos auditivos em trabalhadores expostos ao ruído ocupacional em um estudo com 400 indivíduos verificou que, quanto maior o tempo de exposição, maior o comprometimento dos limiares auditivos obtidos. Na população estudada, 24,75\% dos audiogramas evidenciaram perda auditiva induzida por ruído (8).

A Norma de Higiene Ocupacional (13) da Fundacentro apresenta uma fórmula, para o cálculo da quantidade de pulsos de impacto a que o trabalhador pode ficar exposto em cada jornada de trabalho, de acordo com a magnitude do impulso. A Norma Reguladora 15 (NR-15) estabelece ainda, que o trabalhador não deve ficar exposto a níveis de pressão sonora superiores a $130 \mathrm{~dB}(\mathrm{C})$ (14).

Em um recente estudo sobre a acústica e psicoacústica do ruído das principais armas utilizadas pela polícia militar, foram analisados os disparos do revólver .38 e da pistola .40 (ambos da marca Taurus), com a utilização de decibelímetro digital e análise acústica por meio do software Praat. Os dados foram comparados com os resultados audiométricos de 30 policiais militares portadores de perda auditiva (limiar pior que $25 \mathrm{~dB}$ ). Os picos máximos medidos foram de $113,1 \mathrm{~dB}(\mathrm{C})$ para pistola .40 e $116,8 \mathrm{~dB}(\mathrm{C})$ para revólver 38. Os valores obtidos na análise psicoacústica ficaram entre 17,9 $\pm 0,3$ Barks, correspondendo à faixa entre 4120 e $4580 \mathrm{~Hz}$. As medidas da audiometria indicaram maior perda auditiva na faixa de $4 \mathrm{kHz}$ em $86,7 \%$ dos casos, seguida da frequência de $6 \mathrm{kHz}(66,7 \%)$. Os autores concluíram que a partir da análise acústica dos tiros, foi possível demonstrar causa e efeito entre as principais áreas de excitação de energia na cóclea (cocleograma Praat) e as frequências com diminuição de acuidade auditiva (9).

Durante os treinamentos no estande de tiros, o número de policiais participantes variou entre 12 e 15, e cada indivíduo efetuou 50 disparos. Sendo assim, multiplicando o número de tiros, pelo número de indivíduos, foram calculados de 600 a 750 pulsos de impacto por período de treinamento (9).

Estudos sobre o perfil auditivo em militares realizados no Brasil têm revelado elevado índice de perda auditiva nesta população, fato este associado à exposição excessiva a ruídos de impacto sem o uso de equipamento de proteção individual, uma vez que, a medição do nível de pressão sonora do ruído emitido pelo fuzil automático leve (FAL 7,62 mm) foi de $147 \mathrm{~dB}$ (C) (15).

Uma pesquisa sobre o perfil auditivo de Militares do Exército Brasileiro, realizada por meio de entrevista, otoscopia e exames audiométricos, observou quadro otológico sugestivo de perda auditivia induzida por ruído em $38,1 \%$ dos exames nos militares avaliados. Verificou também que a perda auditiva foi mais intensa quanto maior a idade e o tempo de serviço (6).

Outro estudo avaliou a prevalência do dano auditivo em trabalhadores do setor de manutenção de aeronaves de asas rotativas em uma unidade da Força Aérea Brasileira. Neste estudo foi observada uma prevalência de perda auditiva neurossensorial por exposição continuada a níveis elevados de pressão sonora de 32,4\%, sendo possível identificar como principais fatores relacionados às perdas: o tempo de trabalho e a faixa etária (16).

Relevantes pesquisas na área da saúde auditiva demonstraram correlação estatisticamente significante entre o tempo de exposição ao ruído ocupacional e os limiares auditivos $(6,8,17)$. Nas Forças Armadas de Singapura foi desenvolvido um trabalho para verificar os efeitos do treinamento militar básico na audição. Os autores analisaram as audiometrias de 85 militares antes e depois do treinamento e verificaram uma prevalência de 9,4\% de perda auditiva, que se manteve igual após um ano. O estudo permitiu concluir que o programa de conservação auditiva existente nas Forças Armadas de Singapura é eficiente e resguarda a saúde auditiva de seus militares (18).

Uma importante pesquisa realizou o levantamento do perfil audiológico de cinco instrutores de tiro da polícia 
militar de Montes Claros - MG. Na aferição dos níveis de pressão sonora, o nível médio encontrado no estande de tiro foi de 97,4 dB e dos cinco policiais analisados dois apresentaram curva audiométrica com configuração em entalhe, sendo um unilateral e o outro bilateral, e um apresentou perda auditiva neurossensorial de grau leve nas altas frequências, com configuração da curva audiométrica em gota (19).

$\mathrm{Na}$ França foi realizado um estudo para avaliar o risco de perda auditiva induzida por ruído em policiais. Os resultados demonstraram que os policiais tiveram 1,4 vezes maior probabilidade de apresentarem perda auditiva em relação a servidores civis. Esta probabilidade aumentou para 3 vezes, se considerados os policiais motociclistas. Os autores reforçaram a necessidade de trabalhos com policiais, em virtude da escassez dos mesmos comparados a estudos com soldados (20).

A Prevalência da perda auditiva induzida por ruído entre policiais de trânsito na Cidade Metropolitana Dhaka (Bangladesh), revelou perda auditiva em 24\% dos 100 policiais avaliados, sendo que as frequências mais afetadas foram as de 4 e $6 \mathrm{kHz}$. Em relação à história clínica, 23 indivíduos apresentaram queixa de zumbido e somente 5 apresentaram queixa de disacusia (21).

Na polícia militar do estado de São Pauloé evidente o elevado nível de insalubridade no estande de tiros, entretanto temos que considerar outros setores e/ou serviços da polícia que também podem expor o policial ao risco de perda auditiva, são eles: o Copom (Centro de Operações da Polícia Militar) setor de telefonia disponibilizado pela corporação para o atendimento das emergências policiais e para a coordenação das atividades de policiamento ostensivo; a polícia rodoviária que executa os serviços de fiscalização, policiamento e controle do trânsito e a Rocam (Rondas Ostensivas com Apoio de Motocicletas) que tem tido papel fundamental na segurança do trânsito (22).

O objetivo deste estudo foi pesquisar o perfil audiológico em policiais militares do interior do estado de São Paulo, e correlacionar à idade e o tempo de exposição ocupacional com os achados audiológicos.

\section{MÉTOdo}

No presente estudo foram avaliados 200 policiais militares do $9^{\circ}$ Batalhão da Polícia Militar do Interior - $9^{\circ}$ BPM-I, sendo 169 (84,5\%) do gênero masculino e 31 $(15,5 \%)$ do feminino, com idades entre 25 e 45 anos (média de $38,83 \pm 5,05$ ), com média de tempo de serviço de $16,80 \pm 6,27$ anos.
Os policiais participaram da avaliação audiológica de forma voluntária, foram incluídos no estudo todos os policiais que apresentaram idade dentro da faixa etária proposta.

Os procedimentos utilizados foram: anamnese audiológica (Anexo), otoscopia, audiometria tonal liminar e imitanciometria.

Os exames de audiometria foram realizados em cabina acústica, com o uso de audiômetro GSI 61 Grason Stadler. Para as medidas de imitância acústica foi utilizado o imitanciômetro GSI 38 Grason Stadler.

\section{Anexo. Anamnese Audiológica.}

I. Identificação

Nome:

Data de Nasc: _ I__ Idade:__anos Sexo:() M()F

Data: / / Naturalidade:

2.Queixa:

() Disacusia () Otalgia () Plenitude auricular

()Autofonia ()Recrutamento

()Compreensão de fala diminuída

()Vertigem () Tontura () prurido

() Zumbido () Purgação

() Hábitos Orais: () fumo () álcool

() Otites ()Problemas metabólicos

()Hipertensão arterial（)Ocorrência Familiar

( ) Outros:

Duração e Evolução: Início

() Progressiva () Não-progressiva () Surtos.

Quando:

() Lenta () Rápida

PredominânciaAuditiva:()direita () esquerda

Tratamento: () Médico () Psicológico ()Fonoaudiológico

( ) Outros:

Medicamentos:

3. Dados do Trabalho:

A quanto tempo o(a)senhor(a) trabalha com exposição a ruído

(incluir serviços anteriores): ___ anos ___ meses ( ); anos meses (serviços anteriores)

Duração da Jornada: ___ Horas/Dia; ___ Dias semanais

Setor de trabalho:

Função:

Nível de Ruído exposto no trabalho: $\mathrm{dB}$

Fazuso de E.P.I. (Equipamento de proteção individual):

() Sempre ()Algumas vezes () Quase nunca () Nunca

Qual otipo do protetor auditivo:

() Plugue () Concha/ () lado dir. ()esq.

Realizou repouso auditivo: () Sim () Não

() dir. e esq. Horas 
O presente trabalho contou com a aprovação do Comitê de Ética em Pesquisa (protocolo no 2762/2007).

Os policiais são devidamente equipados com equipamentos de proteção individual (E.P.I.s.), conforme NR 620, da Portaria 3214/78 (14), sendo relevante para o nosso estudo ressaltar a utilização de protetores auriculares (tipo concha), com certificado de aprovação (C.A.) pelo Ministério do Trabalho e Emprego.

No que diz respeito à análise dos dados, foram realizadas: comparação entre os limiares audiométricos, com a idade e o tempo de exposição, foi realizada também a classificação da perda auditiva de acordo com MERLuzzi et al. (23). Para as análises comparativas foi aplicado a Análise de Correlação de Spearman com nível de significância de $5 \%$.

\section{RESULTADOS}

As principais queixas apontadas durante anamnese audiológica foram: zumbido $(\mathrm{n}=52 / 26 \%)$, disacusia $(n=36 / 18 \%)$, plenitude auricular $(n=24 / 12 \%)$ e autofonia $(\mathrm{n}=24 / 12 \%)$.
$\mathrm{Na}$ imitanciometria foram encontradas 100\% de curvas tipo A (24), e houve a presença de recrutamento em 20 (10\%) casos.

No que diz respeito ao resultado das audiometrias, foi observado que na população estudada em 22,75\% $(\mathrm{OD}=21,5 \%$ e $\mathrm{OE}=24 \%)$ dos casos houve perda auditiva induzida por ruído, abaixo se encontra a Tabela 1 com a classificação da perda auditiva, segundo MerLuzzi et al. (23).

Na análise estatística da correlação entre o tempo de exposição no trabalho e os limiares auditivo, foi observado correlação significativa na orelha direita, nas frequências de 1, 3, 4, 6 e $8 \mathrm{kHz}$ e na orelha esquerda nas frequências de 4,6 e $8 \mathrm{kHz}$.

Em relação à correlação entre a idade e a média dos limiares auditivos, foi observada significância estatística em ambas as orelhas a partir da frequência de $2 \mathrm{kHz}$. As Tabelas 2 e 3 apresentam a correlação entre o tempo de exposição e a idade com os limiares auditivos.

A Tabela 4 apresenta a distribuição de alteração audiométrica medida na amostra da população de militares por faixa de tempo de serviço, foram

Tabela I. Classificação dos achados audiométricos de acordo com MerLuZZI et al. (23).

\begin{tabular}{lccccccccc}
\hline & \multicolumn{10}{c}{ Níveis de Perda de Audição } \\
& Normal & 1 & 2 & 3 & 4 & 5 & 6 & 7 & Total \\
\hline $\mathrm{OD}$ & 146 & 24 & 16 & 1 & 2 & 0 & 0 & 11 & 200 \\
$\%$ & 73 & 12 & 8 & 0,5 & 1 & 0 & 0 & 5,5 & 100 \\
$\mathrm{OE}$ & 144 & 22 & 23 & 2 & 1 & 0 & 0 & 8 & 200 \\
$\%$ & 72 & 11 & 11,5 & 1 & 0,5 & 0 & 0 & 4 & 100 \\
\hline
\end{tabular}

Legenda: Os níveis de perda auditiva equivalem a: Níveis de 1 a $5=$ perda de audição induzida por ruído; Nível $6=$ perda de audição mista (ruído + outra causa); Nível 7 = perda de audição de etiologia diversa do ruído.

Tabela 2. Correlação entre o tempo de exposição ocupacional e a média dos limiares auditivos dos 200 policiais militares (Análise de Correlação de Spearman).

\begin{tabular}{lcccc}
\hline Frequência $(\mathrm{Hz})$ & \multicolumn{2}{c}{$r$} & \multicolumn{2}{c}{$P$} \\
& OD & OE & OD & OE \\
\hline 500 & 0,083 & 0,058 & 0,418 & 0,241 \\
1000 & 0,058 & 0,803 & $0,018^{*}$ & 0,415 \\
2000 & 0,064 & 0,056 & 0,432 & 0,369 \\
3000 & 0,137 & 0,175 & $0,013^{*}$ & 0,053 \\
4000 & 0,286 & 0,218 & $0,002 *$ & $<0,001 *$ \\
6000 & 0,224 & 0,231 & $0,001 *$ & $<0,001 *$ \\
8000 & 0,152 & 0,221 & $0,002 *$ & $0,03 I^{*}$ \\
\hline
\end{tabular}

Legenda: OD - orelha direita / OE - orelha esquerda

$\mathrm{r}=$ coeficiente de correlação

$*$ significância para $\mathrm{p}<0,05$
Tabela 3. Correlação entre a idade e a média dos limiares auditivos dos 200 policiais militares (Análise de Correlação de Spearman).

\begin{tabular}{|c|c|c|c|c|}
\hline \multirow[t]{2}{*}{ Frequência $(H z)$} & \multicolumn{2}{|c|}{$r$} & \multicolumn{2}{|c|}{$\mathrm{p}$} \\
\hline & OD & OE & $O D$ & OE \\
\hline 500 & 0,159 & 0,122 & $0,025 *$ & 0,084 \\
\hline 1000 & 0,108 & 0,087 & 0,126 & 0,221 \\
\hline 2000 & 0,153 & 0,187 & $0,03 \mid *$ & $0,008 *$ \\
\hline 3000 & 0,286 & 0,284 & $<0,\left.00\right|^{*}$ & $<0,\left.00\right|^{*}$ \\
\hline 4000 & 0,351 & 0,388 & $<0,\left.00\right|^{*}$ & $<0,\left.00\right|^{*}$ \\
\hline 6000 & 0,352 & 0,308 & $<0,001 *$ & $<0,\left.00\right|^{*}$ \\
\hline 8000 & 0,337 & 0,392 & $<0,001 *$ & $<0,001 *$ \\
\hline
\end{tabular}

Legenda: OD - orelha direita / OE - orelha esquerda

$r=$ coeficiente de correlação

* = significância para $\mathrm{p}<0,05$ 
Tabela 4. Distribuição de alteração audiométrica medida na amostra da população de militares com perda auditiva (limiar maior que $25 \mathrm{~dB}$ ) por faixa de tempo de serviço.

\begin{tabular}{lccccccccc}
\hline$f(\mathrm{kHz})$ & Alteração audiométrica & \multicolumn{4}{c}{ Tempo de Serviço (anos) } & Total & Total & Média OD/OE \\
& & $0-10$ & $11-15$ & $16-20$ & $21-25$ & $26-34$ & $\mathrm{~N}$ & $\%$ & $\%$ \\
\hline$<3,0$ & OD & 0 & 2 & 3 & 2 & 0 & 7 & 12,96 & 17,19 \\
3,0 & OE & 1 & 3 & 3 & 5 & 0 & 12 & 21,42 & \\
& OD & 3 & 3 & 5 & 7 & 2 & 20 & 37,03 & 42,62 \\
4,0 & OE & 5 & 5 & 6 & 8 & 3 & 27 & 48,21 & \\
& OD & 2 & 5 & 6 & 10 & 3 & 26 & 48,15 & 56,22 \\
6,0 & OE & 5 & 5 & 10 & 13 & 3 & 36 & 64,29 & \\
& OD & 2 & 7 & 13 & 8 & 4 & 34 & 62,96 & 67,19 \\
8,0 & OE & 5 & 8 & 9 & 15 & 3 & 40 & 71,42 & \\
& OD & 4 & 6 & 6 & 10 & 6 & 32 & 59,25 & 55,52 \\
& OE & 4 & 6 & 5 & 9 & 5 & 29 & 51,79 & \\
\hline
\end{tabular}

Legenda: OD: orelha direita; OE: orelha esquerda; N: número de orelhas com alteração audiométrica.

consideradas as orelhas com perda auditiva, todos os casos com limiar maior que $25 \mathrm{~dB}$ em uma ou mais frequências do audiograma ( 54 casos na orelha direita e 56 casos na orelha esquerda). Os resultados evidenciaram maior número de casos de perda auditiva nas frequências de $4 \mathrm{kHz}(56,22 \%)$ e $6 \mathrm{kHz}$ $(67,19 \%)$.

\section{DISCUSSÃO}

$\mathrm{Na}$ análise das anamneses as principais queixas apresentadas pela população estudada, zumbido (26\%) e disacusia (18\%), são as mesmas apresentadas como as mais frequentes pela literatura especializada $(9,10)$. Entretanto, um estudo com policiais de trânsito, apresentou uma desproporção entre as queixas de zumbido (24\%) e de disacusia (5\%), possivelmente pelo grau da perda auditiva ter sido leve em $20 \%$ dos casos e moderado em $4 \%$, fato este, que minimizou a queixa de disacusia, frente ao zumbido (21).

Os casos avaliados neste estudo, não apresentaram quaisquer alterações e/ou obstruções do meato acústico externo que pudessem comprometer os resultados da avaliação audiológica. Dessa forma, em todos os casos, foram obtidas curvas timpanométricas do tipo A (24), compatíveis com funcionamento de orelha média normal ou perda auditiva neurossensorial.

Os resultados da audiometria tonal foram classificados de acordo com Meruuzzi et al. (23), e considerando os casos diagnosticados como PAIR, os dados obtidos da orelha direita demonstraram que $43(21,5 \%)$ orelhas apresentaram perda auditiva. Na orelha esquerda foram diagnosticadas 48 (24\%) orelhas com perda. Estes dados foram semelhantes aos obtidos por estudo realizado em ambiente ruidoso industriais (8) e com policiais de trânsito
(21), entretanto comparando os achados com os obtido em militares de carreira, houve maior número de casos com perda nestes últimos $(6,12)$.

O maior número de casos com perda auditiva em militares pode ser explicado em virtude do tipo do armamento pesado utilizado pelo exército, com níveis de ruído superiores a $147 \mathrm{~dB}(\mathrm{C})$ (15). Enquanto que as medições em estande de tiro da policia militar, encontraram valores entre $113,1 \mathrm{~dB}(\mathrm{C})$ para a pistola .40 e $116,8 \mathrm{~dB}(\mathrm{C})$ para o revólver 38 (9).

Considerando a fórmula preconizada pela Norma de Higiene Ocupacional (13) da Fundacentro, o nível máximo de intensidade para uma exposição de ruído de impacto medidos (circuito de compensação C) para 600 pulsos de impacto é de $109 \mathrm{~dB}$. Este dado nos permite identificar que os policiais militares apresentam o ruído de impacto de arma de fogo, como um dos principais elementos danosos para a audição, uma vez que foram medidos valores superiores a 113,1 dB (C), no estande de tiros da polícia militar (9).

Outros elementos insalubres para a saúde auditiva foram identificados na polícia da França e de Bangladesh, sendo estes relacionados ao uso de motocicletas e ao ruído do trânsito $(20,21)$. O uso de motocicletas e a exposição ao ruído do trânsito, também são elementos presentes na atuação da polícia militar do estado de São Paulo (22).

No presente estudo a faixa de frequência com maior percentual de perda auditiva ficou entre 4 e $6 \mathrm{kHz}$, dado este coincidente com a literatura especializada (1), e com estudos realizados em policiais de trânsito na cidade de Dhaka (21). Além disso, um recente estudo demonstrou a relação causa e efeito entre o ruído dos disparos das armas de fogo, e estas áreas com maior lesão na cóclea (9). 
Dentre estas duas frequências ( 4 e $6 \mathrm{kHz}$ ), a que apresentou maior número de casos com alteração no audiograma, em nossos resultados, foi a de $6 \mathrm{kHz}$. Este dado está de acordo com outros estudos feitos com trabalhadores expostos a ruído $(4,5,6)$. Entretanto, não há consenso na literatura em relação à frequência mais acometida, uma vez que, outros estudos descreveram a frequência de $4 \mathrm{kHz}$ com maior percentual de piora na audiometria $(7,8,9)$.

O trabalho revelou ainda que existe correlação significativa entre o aumento da idade e do tempo de serviço, com a piora dos limiares auditivos nas altas frequências em ambas as orelhas. Este dado corrobora com outros estudos relacionados a tempo de exposição e PAIR $(6,8,12,16,17)$.

\section{CONCLUSÃO}

A partir da análise dos dados audiológicos foi possível verificar que os policiais militares são uma população que apresenta risco para desenvolver a perda auditiva. E na comparação entre a idade e o tempo de exposição com os limiares auditivos, houve correlação significativa, evidenciando o perigo da exposição ao ruído. Sendo assim, vemos a necessidade de implementação de programa de conservação auditiva para os policiais militares.

\section{REFERÊNCIAS BIBLIOGRÁFICAS}

1. Jeger S, Jeger J. Alterações auditivas. Um manual para avaliação clínica. São Paulo: Atheneu; 1996. p.133-38.

2. Fiorini AC. Audição: impacto ambiental e ocupacional. Em: Ferreira LP, Befi-Lopes DM, Limongi SCO. Tratado de fonoaudiologia. São Paulo: Roca, 2004, p. 631-42.

3. American College of Occupational and Enviromental Medicine; ACOEM Evidence-Based Statement. Noiseinduced hearing loss. J Occup Environ Med. 2003, 45(6):57981.

4. Ruggieri M, Cattan S, Giardini LDL, Oliveira KAS. Deficiência auditiva induzida por ruído em 472 trabalhadores da região do ABC paulista. Arq Méd ABC. 1991, 14(1):1923.

5. Corrêa Filho HR, Costa LS, Hoehne EL, Pérez MG, Nascimento LCR, Moura EC. Perda auditiva induzida por ruído e hipertensão em condutores de ônibus. Rev Saúde Pública. 2002, 36(6):693-701.

6. Silva AP, Costa EA, Rodrigues SMMR, Souza HLR, Massafera VG. Avaliação do perfil auditivo de militares de um quartel do Exército Brasileiro. Rev Bras Otorrinolaringol. 2004, 70 (3):344-50

7. Araújo SA. Perda auditiva induzida pelo ruído em trabalhadores de matalúrgica. Rev Bras Otorrinolaringol. 2002, 68(1):47-52.

8. Lopes AC, Nelli MP, Lauris JRP, Amorim RB, Melo ADP. Condições de saúde auditiva no trabalho: investigação dos efeitos auditivos em trabalhadores expostos ao ruído ocupacional. Arq Int Otorrionlaringol. 2009, 13 (1):49-54.

9. Guida HL, Kinoshita SK. Diniz TH. Análise acústica e psicoacústica do ruído de armas utilizadas pela polícia militar. Brazilian Journal of Otorhinolaryngology. In press 2010.

10. Dias A, Cordeiro R, Corrente JE, Gonçalves CGO. Associação entre perda auditiva induzida pelo ruído e zumbidos. Cad Saúde Pública. 2006, 22(1):63-8.

11. Caldart AU, Adriano CF, Terruel I, Martins RF, Caldart AU, Mocellin M. Prevalência da perda auditiva induzida pelo ruído em trabalhadores de indústria têxtil. Arq Int Otorrinolaringol. 2006, 10(3):192-196.

12. Ylikoski ME, Ylikoski JS. Hearing loss and handicap of professional soldiers exposed to gunfire noise. Scand. J Work Environ. 1994, 20:93-100.

13. Fundacentro. NHO 01: Norma de higiene ocupacional procedimento técnico - Avaliação da exposição ocupacional ao ruído. [citado 2007 out 31]. Disponível em: http://isegnet.com.br/arquivoscurso/atuais/anexo2.htm

14. Brasil. Portaria no 3214. Ministério do Trabalho 08/07/1978; NR 15. Atividades e operações insalubres (D.O.U. 06/07/1978).

15. Neves BE, Mello MGS. O uso de dispositivos de proteção auditiva nos tiros de fuzil e artilharia. Cad Saúde Colet. 2007, 15:97-116.

16. Ribeiro AMD, Câmara VM. Perda auditiva neurossensorial por exposição continuada a níveis elevados de pressão sonora em trabalhadores de manutenção de aeronaves de asas rotativas. Cad Saúde Pública. 2006, 22:1217-24.

17. Gatto CI, Lemen RA, Teixeira TM, Magni C, Morata TC. A análise da conduta de médicos do trabalho diante de trabalhadores com perda auditiva. Rev Dist Com. 2005, 17(1):101-115.

18. Teo KJ, Chia SE, Tan CT, Ali SM. Effects of basic military training on hearing in the Singapore Armed Forces. Singapore Med. 2008, 49:243-47. 
19. Silva RCL, Zuba DCD. Perfil audiológico dos instrutores de tiro da polícia militar de Montes Claros - MG. Rev Consciência Extensão. 2008, 1:14-24.

20. Lesage FX, Jovenin N, Deschamps F, Vincent S. Noiseinduced hearing loss in French police officers. Occupational Medicine, 2009, 3:1-4.

21. Sharif A, Taous a, Siddique BH, Dutta PG. Prevalence of noise induced hearing loss among traffic police in Dhaka Metropolitan City. Mymensingh Med J. 2009, 18:24-28.
22. Polícia Militar do Estado de São Paulo. Secretaria do Estado dos Negócios de Segurança Pública. [citado em 2010 ago 27]. Disponível em: http://www.polmil.sp.gov.br/inicial.asp

23. Merluzzi F, et al. Metodologia di esecuzione del controllo dell'udito dei lavoratori esposti a rumore. Nuovo Arch Ital Otol. 1979, 7:695-714.

24. Jerger J. Clinical experience with impedance audiometry. Arch Otolaryngol. 1970, 92(4):311-24. 